This document is the accepted manuscript version of the following article:

Dünki, S. J., Tress, M., Kremer, F., Ko, S. Y., Nüesch, F. A., Varganici, C. -D., ... Opris, D. M. (2015). Fine-tuning of the dielectric properties of polysiloxanes by chemical modification. RSC Advances, 5(62), 50054-50062.

http://doi.org/10.1039/C5RA07412F

ARTICLE

\title{
Fine-tuning of the dielectric properties of polysiloxanes by chemical modification
}

\author{
Simon J. Dünki, ${ }^{a, b}$ Martin Tress, ${ }^{c}$ Friedrich Kremer, ${ }^{c} *$ Song Yee Ko, ${ }^{a, b}$ Frank A. Nüesch, ${ }^{a, b}$ Cristian- \\ Dragos Varganici, ${ }^{d}$ Carmen Racles, ${ }^{d}$ Dorina M. Opris ${ }^{a} *$
}

\begin{abstract}
A series of novel polysiloxanes is presented the glass transition temperatures and dielectric properties of which are systematically fine-tuned by utilizing thiol-ene post-polymerization reactions. The pendant vinyl groups of a high molecular weight polymethylvinylsiloxane P1 were exhaustively reacted with the thiol compounds 1-butanethiol (2) and 3mercaptopropionitrile $\mathbf{3}$ both separately to give polymers $\mathbf{P} \mathbf{2}$ and $\mathbf{P} \mathbf{3}$, respectively, as well as in various ratios $\mathrm{x}, \mathrm{y}$ so as to create materials $\mathbf{P} \mathbf{2}_{\mathbf{x}} \mathbf{P} \mathbf{3}_{\mathbf{y}}$ with greatly differing contents of the polar nitrile group (y). All modifications proceeded quantitatively as was confirmed by ${ }^{1} \mathrm{H}$ NMR spectroscopy. Because of the presence of the polarizable thioether and nitrile groups, the resulting siloxane polymers exhibit permittivity ranging from $\varepsilon^{\prime}=4.7$ to 18.4 for $\mathbf{P} 2$ and $\mathbf{P} 3$, respectively. The $\varepsilon^{\prime}$ values of all polymers $\mathbf{P} \mathbf{2}_{\mathbf{x}} \mathbf{P} \mathbf{3}_{\mathbf{y}}$ carrying more than one kind of thiolether group lie within this range. Additionally, broadband dielectric spectroscopy measurements of $\mathbf{P} \mathbf{2}, \mathbf{P} \mathbf{2}_{\mathbf{1}} \mathbf{P} \mathbf{3}_{\mathbf{1}}$ and $\mathbf{P} \mathbf{3}$ have been conducted in the temperature range from $-150{ }^{\circ} \mathrm{C}$ to $60{ }^{\circ} \mathrm{C}$ and the frequency range from $0.1 \mathrm{~Hz}$ to $1 \mathrm{MHz}$. Due to their high permittivity, polymers $\mathbf{P 2}, \mathbf{P} \mathbf{2}_{\mathrm{x}} \mathbf{P} \mathbf{3}_{\mathbf{y}}$ and $\mathbf{P 3}$ are attractive candidates for dielectric elastomer actuators and flexible electronics.
\end{abstract}

\section{Introduction}

Elastomers are chemically or physically cross-linked polymers that have low calorimetric glass transition temperatures $\left(T_{g}\right)$, show large strains and reversible deformations. ${ }^{1}$ Their mechanical properties can be tuned all the way from soft to stiff by changing the cross-link density from low to high. There is an increasing need for elastomers that have a high dielectric permittivity $\left(\varepsilon^{\prime}\right)$. Such materials are attractive for many fields of applications including flexible electronics, actuators, energy harvesting, and artificial muscles ${ }^{2}$ and are created by either blending or chemical modification. ${ }^{3}$ It has been shown that polarizable fillers can increase the $\varepsilon^{\prime}$, however, such blending approaches often lead to worsened elastic properties. $^{4}$

\footnotetext{
a. Empa, Swiss Federal Laboratories for Materials Science and Technology, Laboratory for Functional Polymers, Ueberlandstr. 129, CH-8600, Dübendorf, Switzerland, E-mail : dorina.opris@empa.ch.

${ }^{b .}$ Ecole Polytechnique Fédérale de Lausanne (EPFL), Institut des matériaux, Station 12, CH 1015, Lausanne, Switzerland.

Institute of Experimental Physics I, University of Leipzig, Linnéstr. 5, 04103 Leipzig, Germany, E-mail: friedrich.kremer@physik.uni-leipzig.de.

d. Petru Poni Institute of Macromolecular Chemistry, Aleea Grigore Ghica Voda 41A, Iasi, 700487, Romania

Electronic Supplementary Information (ESI) available: ${ }^{1} \mathrm{H}$ and ${ }^{13} \mathrm{C}$ NMR, GPC, TGA of polymers $\mathbf{P}_{\mathbf{2}}, \mathbf{P}_{\mathbf{3}}$, and $\mathbf{P} \mathbf{2}_{\mathbf{x}} \mathbf{P} \mathbf{3}_{\mathbf{y}}, 2 \mathrm{D}$ NMR spectra for $\mathbf{P} \mathbf{2}_{\mathbf{1}} \mathbf{P} \mathbf{3}_{\mathbf{1}}, \mathrm{DMA}$ and stress-strain curve of a material prepared by cross-linking $\mathbf{P} \mathbf{3}$, dielectric properties at different temperatures for $\mathbf{P}_{3}$, and $\mathbf{P} \mathbf{2}_{x} \mathbf{P} \mathbf{3}_{y}$, and dielectric properties at room temperature as function of frequency for polymers $\mathbf{P}_{\mathbf{2}}, \mathbf{P}_{\mathbf{3}}$, and $\mathbf{P} \mathbf{2}_{\mathbf{x}} \mathbf{P} \mathbf{3}_{\mathbf{y}}$. Supporting Information is available from the Wiley Online Library or from the author. See DOI: $10.1039 / x 0 \times x 00000 x$
}

Permittivity can also be increased by introducing polar groups through chemical modification of the polymeric material. This approach has the downside that it often leads to an undesired increase of $T_{g}$ which detrimentally impacts the elastic properties. ${ }^{5}$ Thus, the chemical modification approach requires subsequent measures to lower the $T_{g}$ of the initially obtained polar polymer. That this strategy can be successful is illustrated by metathesis polymers with fluorinated side chains. ${ }^{6}$ While their $T_{g}$ 's were unattractively high, hydrogenation of the polymers resulted in a product with a $40{ }^{\circ} \mathrm{C}$ lower $T_{g}$. Hydrogenation converts unsaturated backbone units into saturated ones and therefore increases backbone flexibility. Plasticisers have also been used to decrease $T_{g}{ }^{7}$ Even rigid plastics such as poly(vinyl chloride) can be made flexible when plasticizers are added. ${ }^{8}$

Inspired by the work of Feast et al., ${ }^{9}$ a different approach was recently suggested. ${ }^{10}$ The idea was to start from a polymer with such a low $T_{g}$ that in spite of the inherent increase in $T_{g}$ by the introduction of polar groups, the $T_{g}$ of the product would still be attractively low. Polysiloxanes show the lowest $T_{g}$ among polymers, are easily accessible and cheap, and allow chemical functionalization. This makes them attractive candidates for such endeavour. Meanwhile, several groups have reported on polysiloxanes modified with polar groups which showed increased permittivity. For example, small dipoles like nitrile, chloro, and trifluoropropyl as well as large dipoles such as 4-nitroaniline and 4nitrobenzene, have been used. ${ }^{11}$ The functionalization is done either by using a hydrosilylation reaction ${ }^{12}$ or an azide-alkyne Huisgen cycloaddition. ${ }^{13}$ The attachment of the polar groups via a 
Pt-catalysed hydrosilylation reaction between the $\mathrm{Si}-\mathrm{H}$ bonds of the polymer chain and the vinyl group present in the polar entity turned out to be non-quantitative resulting in a tendency for unwanted cross-linking through the residual $\mathrm{Si}-\mathrm{H}$ bonds. ${ }^{14}$ This reaction is also sensitive towards various impurities such as thiols and amines which can poison the rather expensive Pt catalyst. Finally, this chemistry is problematic whenever materials are concerned where residual traces of $\mathrm{Pt}$ are not acceptable. The azide-alkyne cycloaddition has been extensively used for the functionalization of polymers. ${ }^{15}$ This reaction was recently used to attach the polar nitrobenzene to polysiloxanes containing azide pendant groups and polymers with increased permittivity were obtained. ${ }^{16}$

Despite the progress achieved in making functional polysiloxanes with increased $\varepsilon^{\prime}$ accessible, the molecular weights of these polymers were too low to allow their cross-linking into robust elastomeric materials with large strain at break. At this point the present work sets in. It opens access to high molecular weight functionalized polysiloxanes with increased permittivity and overcomes the above mentioned drawbacks by combining a more robust reaction for the chemical functionalization with the avoidance of a heavy metal catalyst. We here report a series of new polysiloxanes containing varying amounts of polar nitrile groups starting from polymethylvinylsiloxane (P1) via a thiol-ene addition. The nitrile functional group was selected because of its polarity and low volume, and therefore it should be easy to polarize by an electric field. The pendant vinyl groups of a high molecular weight P1 were exhaustively reacted with the thiol compounds 1butanethiol (2) and 3-mercaptopropionitrile 3 both separately to give polymers $\mathbf{P} \mathbf{2}$ and $\mathbf{P} \mathbf{3}$, respectively, as well as in various ratios $x, y$ so as to create polymers $\mathbf{P} \mathbf{2}_{\mathbf{x}} \mathbf{P} \mathbf{3}_{\mathbf{y}}$ with greatly differing contents of the polar nitrile group (y). These polymers were characterized by ${ }^{1} \mathrm{H}$ NMR, ${ }^{13}$ C NMR, FTIR, GPC, and EA. Additionally, the dielectric properties of these polymers were investigated as a function of dipole concentration. Finally, broadband dielectric spectroscopy measurements on selected samples were conducted at temperatures ranging from $-150{ }^{\circ} \mathrm{C}$ to $+60{ }^{\circ} \mathrm{C}$ and frequencies between $0.1 \mathrm{~Hz}$ to $1 \mathrm{MHz}$. Preliminary experiments show that it is possible to cross-link these polymers to robust elastomers.

\section{Results and discussion}

A series of hydroxyl end-functionalized siloxane polymers P2, P3 and $\mathbf{P} \mathbf{2}_{\mathbf{x}} \mathbf{P} \mathbf{3}_{\mathbf{y}}$ that have nitrile pendant groups was prepared starting from P1 as shown in Scheme 1. For reasons that were outlined in the introduction it was important to start the synthesis from a siloxane polymer that combines high molecular weight with a large concentration of lateral vinyl groups for post-polymerization modification. Since such siloxanes are not commercially available, P1 was synthesized starting from $\mathbf{V}_{\mathbf{4}}$ under anionic polymerization conditions. The thermally labile tetramethylammonium hydroxide (TMAH) initiator was used because the purification of the highly viscous polymer is easily performed by heating above $140^{\circ} \mathrm{C}$. At this temperature the TMAH is decomposing to volatile inactive compounds (triethylamine and methanol) that can be removed together with residual cyclic siloxanes by high vacuum distillation. This reaction gave the starting polymer $\mathbf{P} \mathbf{1}$ in $93 \%$ yield as a highly viscous liquid. P1 was analyzed by ${ }^{1} \mathrm{H}$ NMR, ${ }^{13} \mathrm{C}$ NMR, IR and GPC. The molecular weight of the resulting polymer and its distribution were determined as $M_{n}=72 \mathrm{kDa}, M_{w}=169 \mathrm{kDa}, \mathrm{D}=2.4$ by GPC using polydimethylsiloxane (PDMS) standards. P1 was also prepared in $100 \mathrm{~g}$ scale and it was always this very batch that was used for subsequent functionalization. Using the exact same batch of starting material minimized the influence of end-groups or molecular weight on the permittivity.

For the post-polymerization, a wide range of polar thiols can in principle be used. We focused on $\mathbf{3}$ because it is easily accessible in one step by addition of in-situ generated $\mathrm{H}_{2} \mathrm{~S}$ to acrylonitrile in $60 \%$ yield. Additionally, the nitrile group has a large dipole moment and a rather low volume and it should therefore be easy to polarize. While the click reaction to pendant vinyl groups of polymers has been reported in the literature, preliminary tests were done to confirm that this reaction also proceeds extremely well for our starting materials. ${ }^{19}$ For the thiol-ene addition thermo- or photo- labile radical initiators can be used. Reaction of P1 with $\mathbf{3}$ using azobisisobutyronitrile (AIBN) in refluxing THF was not completed after $3.5 \mathrm{~h}$ whereas the reaction with the photo sensitive radical initiator 2,2-dimethoxy-2-phenylacetophenone (DMPA) was completed in less than five minutes. We therefore focussed on the photo-induced addition which turned out to be fast and reliable. After these orienting studies had been completed, starting material $\mathbf{P} \mathbf{1}$ was reacted with thiols $\mathbf{2 ,} \mathbf{3}$ and various ratios of $\mathbf{2 / 3}$. To avoid uncontrolled cross-linking between the vinyl groups of the siloxane polymers, an excess of thiols to the vinyl groups was used. Total conversion of the vinyl groups was obtained within 5 min (proved by ${ }^{1} \mathrm{H}$ NMR). This allowed us preparation of a series of polymers $\mathbf{P 2}$, $\mathbf{P} \mathbf{3}$ and $\mathbf{P} \mathbf{2}_{\mathbf{x}} \mathbf{P} \mathbf{3}_{\mathbf{y}}$ with different contents of polar nitrile group (Scheme $1)$.

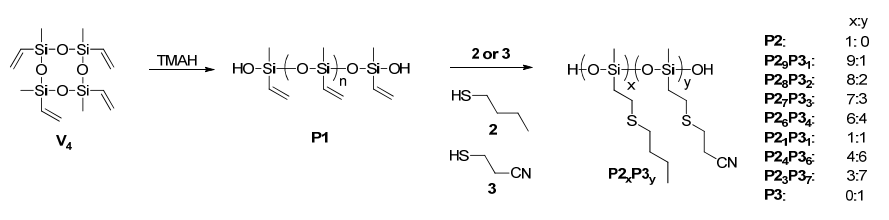

Scheme 1 Synthesis of starting polysiloxane $\mathrm{P} 1$ from monomer $\mathrm{V}_{4}$ and the post-polymerization modification of $\mathrm{P} 1$ with thiols 2 and 3 to polymers $\mathrm{P} 2$ and $\mathrm{P} 3$ and to polymers $\mathrm{P} 2_{\mathrm{x}} \mathrm{P} 3_{\mathrm{y}}$ in which varying ratios of 2 and 3 were incorporated.

To remove the excess of unreacted thiols, first the THF was partially removed and the polymer was precipitated with methanol. The crude polymer was repeatedly dissolved in toluene and precipitated with methanol, until the excess of thiol and other impurities were removed. Fig. 1 shows the ${ }^{1} \mathrm{H}$ NMR spectra of $\mathbf{P 1}, \mathbf{P 2}, \mathbf{P} 3$, and $\mathbf{P} \mathbf{2}_{1} \mathbf{P} \mathbf{3}_{1}$. The disappearance of the vinyl signals of $\mathbf{P} \mathbf{1}$ at about $\delta=6$ ppm and the appearance of new signals in the aliphatic part of the spectra of $\mathbf{P} \mathbf{2}, \mathbf{P} \mathbf{3}$ and $\mathbf{P} \mathbf{2}_{\mathbf{x}} \mathbf{P} \mathbf{3}_{\mathbf{y}}$ due to the addition of the thiols to the vinyl groups are clear proofs of successful coupling (Fig. S1). In addition to this, the disappearance of the vinyl carbons at $\delta=136.7$ ppm and $\delta=133.1 \mathrm{ppm}$ in the ${ }^{13} \mathrm{C}$ NMR spectra is noted as is the appearance of a new signal at $\delta=118.6 \mathrm{ppm}$ for the polymers containing CN groups (Fig. S2 and S3). The proposed structures are further supported by new signals in the aliphatic part of the ${ }^{13} \mathrm{C}$ 
NMR spectra, which were assigned for sample $\mathrm{P} 2{ }_{1} \mathrm{P} 3{ }_{1}$ using $2 \mathrm{D}$ NMR techniques (Fig. S4). In line with a full conversion of the vinyl groups the IR vibration bands at $1406 \mathrm{~cm}^{-1}$ (=C-H in-plane bending), 1600 $\mathrm{cm}^{-1}$ ( $\mathrm{C}=\mathrm{C}$ stretching), and at $3055 \mathrm{~cm}^{-1}$ (=C-H stretching) are absent in all products (Fig. S5). For the polymers $\mathbf{P} \mathbf{3}$ and $\mathbf{P} \mathbf{2}_{\mathbf{x}} \mathbf{P} \mathbf{3}_{\mathbf{y}}$ that contain nitrile groups, the characteristic signal at $2250 \mathrm{~cm}^{-1}$ is present. The content of the nitrile groups in the final copolymers was determined by comparing the integrals of the protons of $\mathrm{Si}-\mathrm{CH}_{3}$ groups at $\delta=0.15 \mathrm{ppm}$ with the integrals of the $6 \mathrm{CH}_{2}$ protons of the addition product of $\mathbf{3}$ to the vinyl groups which appeared between $\delta=2.95 \mathrm{ppm}$ and $\delta=2.62 \mathrm{ppm}$. Fortunately, the reactivity of the two thiols used is similar, and therefore the amount of the nitrile groups incorporated into copolymers was as predicted. The ${ }^{1} \mathrm{H}$ NMR spectra of all prepared copolymers are displayed in the supporting information. Typical elugrams of $\mathbf{P} \mathbf{2}, \mathbf{P} \mathbf{3}$ and $\mathbf{P} \mathbf{2}_{\mathbf{x}} \mathbf{P} \mathbf{3}_{\mathbf{y}}$ are shown in supporting information and the $M_{w}, M_{n}$ and $P D I$ are given in Table 2. The functionalized polymers are shifted to smaller elution volumes compared to $\mathbf{P} \mathbf{1}$. All polymers contained a small fraction of a higher molecular weight $(<3 \%)$ that might form due to a side reaction of the vinyl groups or a condensation of the hydroxyl-end groups.

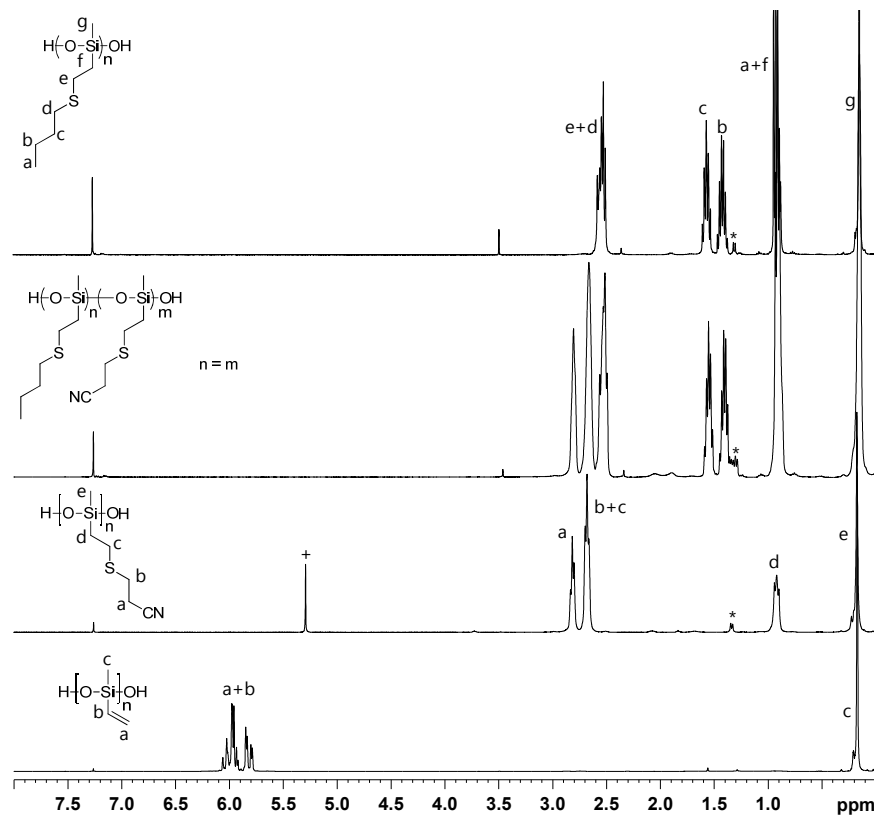

Fig. $1{ }^{1} \mathrm{H}$ NMR spectra of starting $\mathbf{P} \mathbf{1}$ and of the thiol-ene addition products P2, P3 and $\mathbf{P 2} \mathbf{P}_{\mathbf{1}} \mathbf{P} \mathbf{3}_{1}$, dichloromethane $(+)$, methanol $(\times)$, Markovnicov product $(*)$ in $\mathrm{CDCl}_{3}$.

Differential scanning calorimetry (DSC) on $\mathbf{P 1}, \mathbf{P 2}, \mathbf{P} \mathbf{3}$ and $\mathbf{P} \mathbf{2}_{\mathbf{x}} \mathbf{P} \mathbf{3}_{\mathbf{y}}$ was also performed. The samples were heated at a rate of $10{ }^{\circ} \mathrm{C}$ per min from $-150{ }^{\circ} \mathrm{C}$ to $50^{\circ} \mathrm{C}$ and only the second heating curves were taken into account for $T_{g}$ analysis (Fig. 2). For all polymers the $T_{g}$ was well below room temperature. A steady increase in $T_{g}$ with increasing proportion of nitrile groups was observed (Fig. S6). The starting polymer $\mathbf{P} \mathbf{1}$ has a $T_{g}=-128{ }^{\circ} \mathrm{C}$, polymer $\mathbf{P 2}$ without nitrile has a $T_{g}=-97.6{ }^{\circ} \mathrm{C}$ while $\mathbf{P 3}$, with the maximum content of nitrile has a $T_{g}=-52.6{ }^{\circ} \mathrm{C}$. Furthermore, a tiny melting peak was observed in the temperature range between 0 and $25^{\circ} \mathrm{C}$ (Fig. S7) which might indicate that to a very small extent these polymers might be semicrystalline, as it is well established for PDMS. ${ }^{20}$

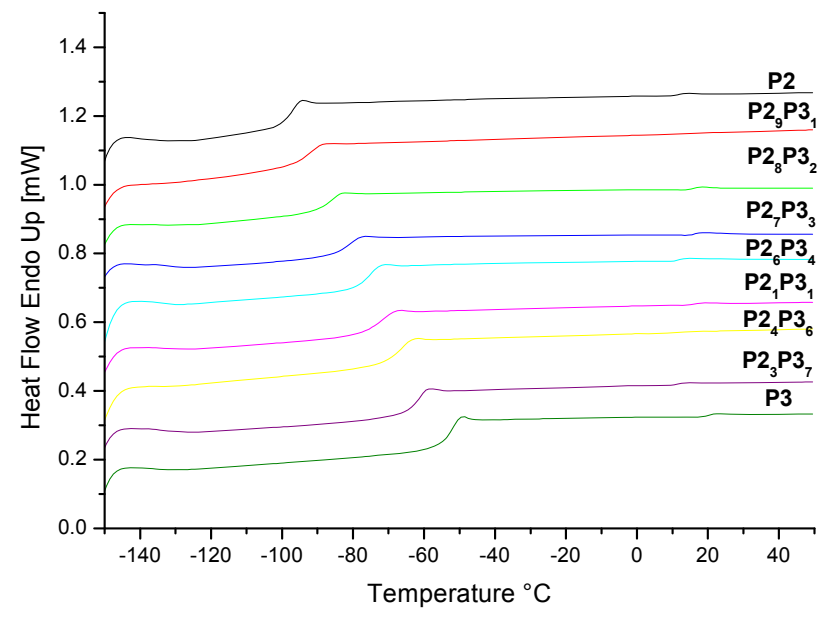

Fig. 2 Variation of glass transition temperature $\left(T_{g}\right)$ with the content of polar groups of $\mathbf{P 2}, \mathbf{P} \mathbf{3}$ and $\mathbf{P} \mathbf{2}_{\mathbf{x}} \mathbf{P} \mathbf{3}_{\mathbf{y}}$.

TGAs recorded under He flow show that all polymers $\mathbf{P 2}, \mathbf{P} 3$ and $\mathbf{P} \mathbf{2}_{\mathbf{x}} \mathbf{P} \mathbf{3}_{\mathbf{y}}$ are stable up to $300{ }^{\circ} \mathrm{C}$ where decomposition starts (with a weight loss between 70 to $75 \%$ ). A negligible influence of the polar nitrile groups on the stability of polymers was observed, e.g. the maximum decomposition temperature for $\mathbf{P 2}$ was $400{ }^{\circ} \mathrm{C}$ while for P3 was $384^{\circ} \mathrm{C}$ (Fig. S8).

To identify the type of fluctuating units in these polymers and to elucidate systematic changes in the dynamics (as indicated by the change in $T_{g}$ ), temperature dependent BDS measurements of the samples $\mathbf{P} \mathbf{2}, \mathbf{P} \mathbf{2}_{\mathbf{1}} \mathbf{P} \mathbf{3}_{\mathbf{1}}$ and $\mathbf{P} \mathbf{3}$ were carried out. Due to the presence of some residual methanol in the polymers (see Table 2), the samples were annealed in dry nitrogen atmosphere for several hours until the remaining solvent evaporated and no change in the dielectric spectra was observed. Fig. 3 shows the dielectric permittivity and loss $\varepsilon^{\prime}$ and $\varepsilon^{\prime \prime}$ as well as the real and imaginary part of the complex conductivity $\sigma^{\prime}$ and $\sigma^{\prime \prime}$ for the sample P2. The dielectric loss is characterized by several relaxation processes in the temperature range between -40 and $-150{ }^{\circ} \mathrm{C}$. Further, at temperatures above -60 ${ }^{\circ} \mathrm{C}$ a clear conductivity contribution is present in the dielectric loss (increase at low frequencies with a slope of -1 ) as well as in the real part of the complex conductivity (plateau). This contribution gives rise to electrode polarization as indicated by the steep increase in the dielectric permittivity as well as the development of a maximum in the imaginary part of the complex conductivity both occurring at low frequencies in the temperature range above $20^{\circ} \mathrm{C}$. By fitting Havriliak-Negami ${ }^{21}$ functions to the dielectric loss spectra (Fig. S9), the mean relaxation times $\tau$ of the underlying structural relaxations are extracted. 


\section{Journal Name}

\section{ARTICLE}

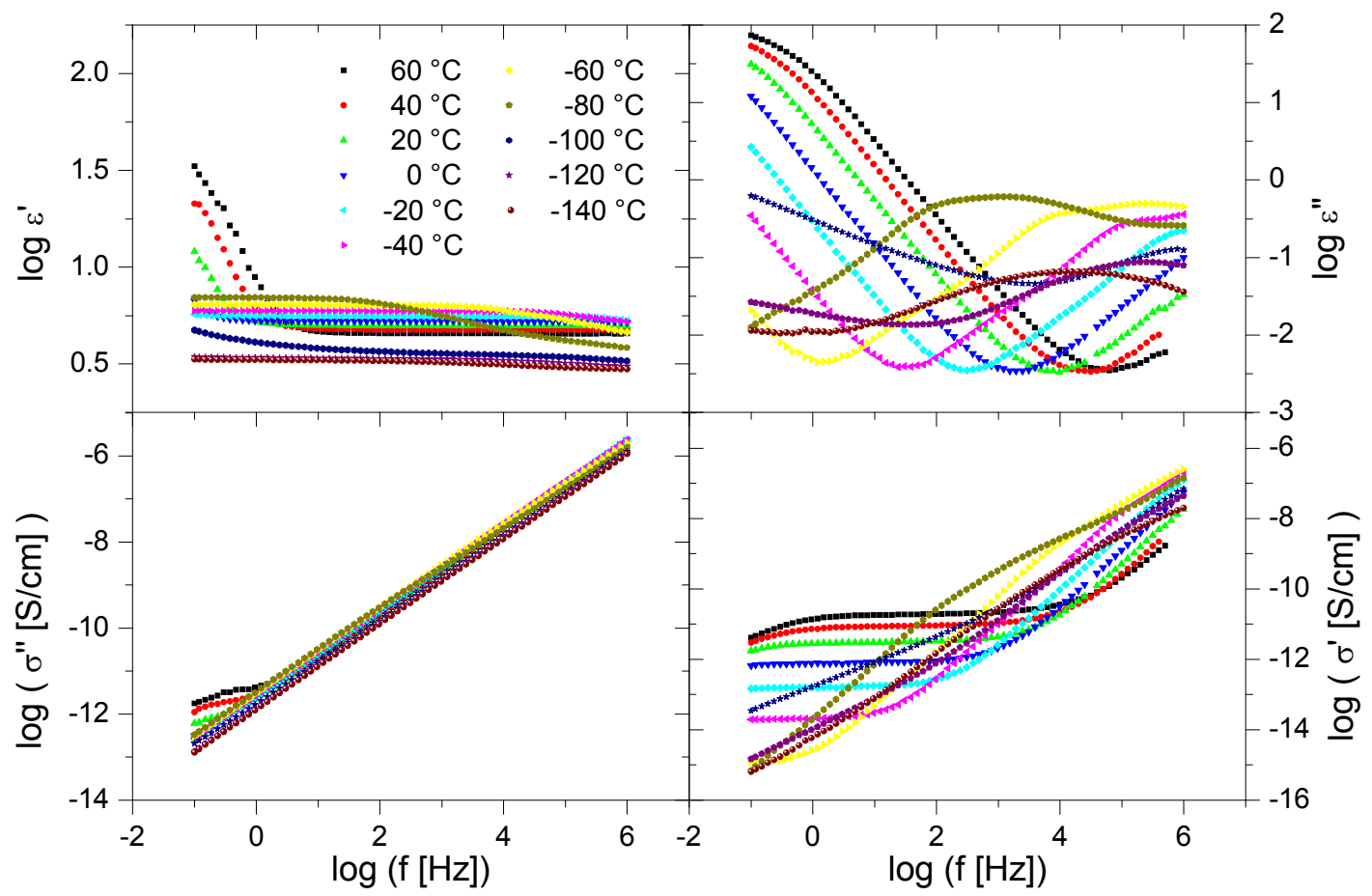

Fig. 3 Dielectric permittivity $\left(\varepsilon^{\prime}\right)$ and loss $\left(\varepsilon^{\prime \prime}\right)$ as well as the real and imaginary part of the complex conductivity $\left(\sigma^{\prime}\right.$ and $\sigma^{\prime \prime}$, respectively) of the sample $\mathbf{P 2}$ as a function of frequency for different temperatures as indicated.

$\mathrm{T}\left[{ }^{\circ} \mathrm{C}\right]$

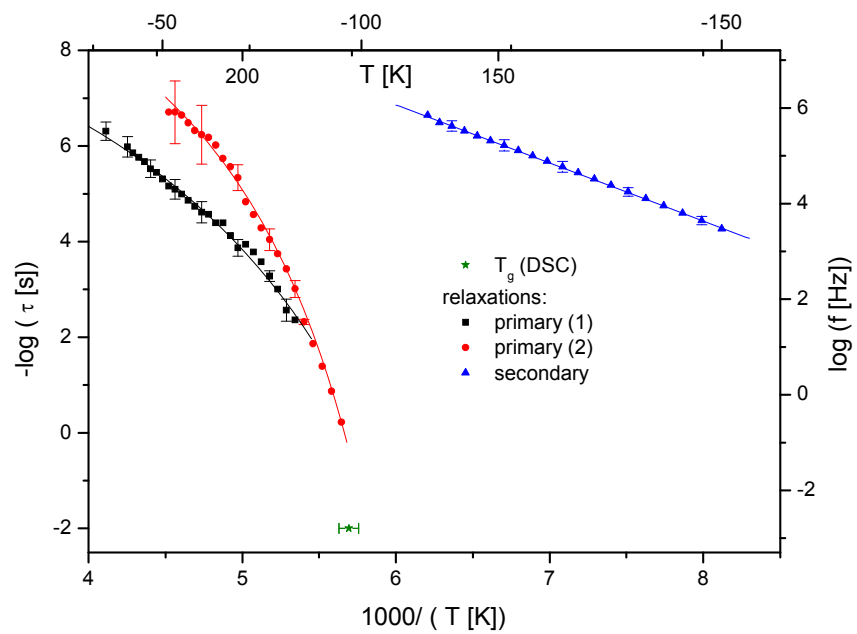

Fig. 4 Activation plot of the mean relaxation times $\tau$ extracted from the dielectric loss spectra (Fig. 3) of sample $\mathbf{P 2}$. Two primary relaxations can be identified according to their Vogel-Fulcher-Tammann temperature dependence, which coincide with the calorimetric glass transition as determined by DSC, as well as a secondary relaxation which exhibits an Arrhenius type activation. The error bars indicate the uncertainty of the fit parameters; in the primary relaxations, this uncertainty is larger due to the proximity of these two processes.
Fig. 4 displays these relaxation times as a function of inverse temperature to identify the type of thermal activation. Two of the processes can be described by a Vogel-Fulcher-Tammann (VFT) equation ${ }^{22}$ typical for the primary relaxation of glassy materials while a third process shows an Arrhenius-type activation which indicates secondary relaxations (see Supp. Inform.). The primary relaxations merge with decreasing temperature and coincide with the calorimetric glass transition temperature as determined by DSC.

The samples $\mathbf{P} \mathbf{2}_{\mathbf{1}} \mathbf{P} \mathbf{3}_{1}$ and $\mathbf{P} \mathbf{3}$ show similar features: two merging primary relaxations, a conductivity contribution with indications for electrode polarization and a secondary relaxation, but at different temperatures (Fig. S10-S13). Beyond this, sample $\mathbf{P} \mathbf{2}_{\mathbf{1}} \mathbf{P} \mathbf{3}_{\mathbf{1}}$ exhibits a further secondary relaxation. In the following, these contributions are analyzed separately to compare the different temperature dependencies in the three samples.

The inset of Fig. 5 displays the mean relaxation times of the primary relaxations of $\mathbf{P 2}, \quad \mathbf{P 2}_{\mathbf{1}} \mathbf{P \mathbf { B } _ { \mathbf { 1 } }}$ and $\mathbf{P} \mathbf{3}$ as a function of inverse temperature as well as the respective calorimetric glass transition temperatures. From sample $\mathbf{P 2}$ over $\mathbf{P} \mathbf{2}_{\mathbf{1}} \mathbf{P} \mathbf{3}_{1}$ to $\mathbf{P 3}$ (i.e. with increasing content of $\mathrm{CN}$ dipoles) the activation curves as well as the $T_{g}$ values shift to higher temperatures. A scaling with respect to the calorimetric $T_{g}$ results in coinciding curves (Fig. 5) indicating a common structural origin of these relaxations. In the rescaled 
graph, also the relaxations of a pure PDMS sample are shown; the faster of the two observed PDMS relaxations coincides with the faster relaxation of the polymers $\mathbf{P} \mathbf{2}, \mathbf{P} \mathbf{2}_{\mathbf{1}} \mathbf{P} \mathbf{3}_{\mathbf{1}}$ and $\mathbf{P} \mathbf{3}$ while the other one is slower by about one order of magnitude compared to $\mathbf{P 2}$, $\mathbf{P} \mathbf{2}_{1} \mathbf{P} \mathbf{3}_{1}$ and $\mathbf{P 3}$. For PDMS, the faster relaxation has been assigned to the segmental motion of the backbone (the structural relaxation underlying glassy dynamics) in the amorphous phase while the slower relaxation is identified with the fluctuation of those segments neighbouring crystalline domains. ${ }^{20}$ Therefore, we attribute the faster primary relaxation to fluctuation of the backbone segments. In contrast, the origin of the slower process in the polymers $\mathbf{P} \mathbf{2}, \mathbf{P} \mathbf{2}_{1} \mathbf{P} \mathbf{3}_{1}$ and $\mathbf{P} \mathbf{3}$ remains unclear so far. Since the temperature dependence of the slow relaxation process is comparable to that one observed in PDMS (merging of the two relaxations with decreasing temperature) a relation to crystalline domains could be suspected. However, the melting peaks observed in the DSC (Fig. S7) indicate a much smaller degree of crystallinity than required to explain the dielectric strength of the slow relaxation. Also a potential mesophase separation (into siloxane and alkyl domains) seems to be an unlikely cause since one would expect the dynamics of such domains to merge with increasing temperature while the contrary is observed. Further investigations are on the way to clarify the origin of this relaxation process.

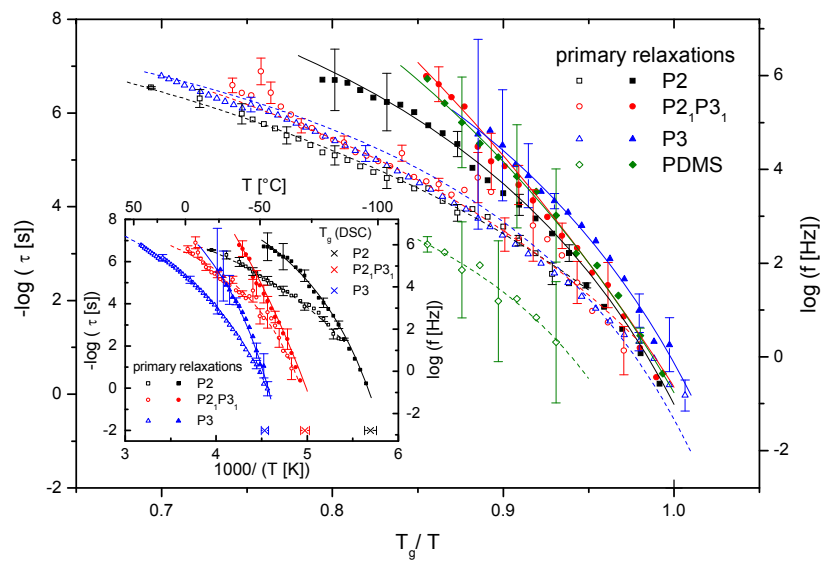

Fig. 5 Mean relaxation time $\tau$ of the primary relaxations of the polymers $\mathbf{P 2}$, $\mathbf{P} \mathbf{2}_{1} \mathbf{P} \mathbf{3}_{1}$ and $\mathbf{P} \mathbf{3}$ as a function of absolute inverse temperature (inset) and rescaled with respect to the corresponding calorimetric glass transition temperature $T_{g}$ (main frame). The error bars indicate the uncertainty of the fit parameters; in the fast primary relaxation of P3 at higher temperatures, this uncertainty increases due to the increasing proximity of the secondary relaxation. Solid and dashed lines represent fits of the Vogel-Fulcher-Tammann equation to the data of the $\alpha$-relaxation in the amorphous phase (solid symbols) and the slow relaxation of yet unexplained origin (open symbols), respectively.

Fig. 6 depicts the thermal activation of the secondary relaxations of the polymers $\mathbf{P} \mathbf{2}, \mathbf{P} \mathbf{2}_{\mathbf{1}} \mathbf{P} \mathbf{3}_{\mathbf{1}}$ and $\mathbf{P} \mathbf{3}$. While $\mathbf{P} \mathbf{2}$ and $\mathbf{P} \mathbf{3}$ show only one secondary relaxation each with activation energies differing by a factor of about $2\left(E_{A}=23.2 \pm 0.1 \mathrm{~kJ} / \mathrm{mol}\right.$ and $E_{A}=51.8 \pm 0.3 \mathrm{~kJ} / \mathrm{mol}$, respectively), the sample $\mathbf{P} \mathbf{2}_{\mathbf{1}} \mathbf{P} \mathbf{3}_{\mathbf{1}}$ exhibits two secondary relaxations which coincide with those of the former polymers within the margins of experimental accuracy. The activation energies of these two are $\mathrm{E}_{\mathrm{A}}=27.0 \pm 0.7 \mathrm{~kJ} / \mathrm{mol}$ and $\mathrm{E}_{\mathrm{A}}=55 \pm 3 \mathrm{~kJ} / \mathrm{mol}$ ).

In polymers, secondary relaxations are typically assigned to local fluctuations of the side chains. Since the side chains of $\mathbf{P 2}$ and $\mathbf{P 3}$ contain different end groups, a difference in the dynamics is anticipated. In contrast to the methyl end groups in $\mathbf{P 2}$, the $\mathrm{CN}$ terminated side chains in $\mathbf{P 3}$ are expected to establish dipolar interactions which slow down the dynamics. This is in accord with the secondary relaxation in $\mathbf{P} \mathbf{3}$ being slower than that one in $\mathbf{P 2}$ as well as its larger activation energy. Furthermore, this interpretation is confirmed by the presence of two relaxations in $\mathbf{P} \mathbf{2}_{\mathbf{1}} \mathbf{P} \mathbf{3}_{\mathbf{1}}$ which contains both types of end groups at the side chains.

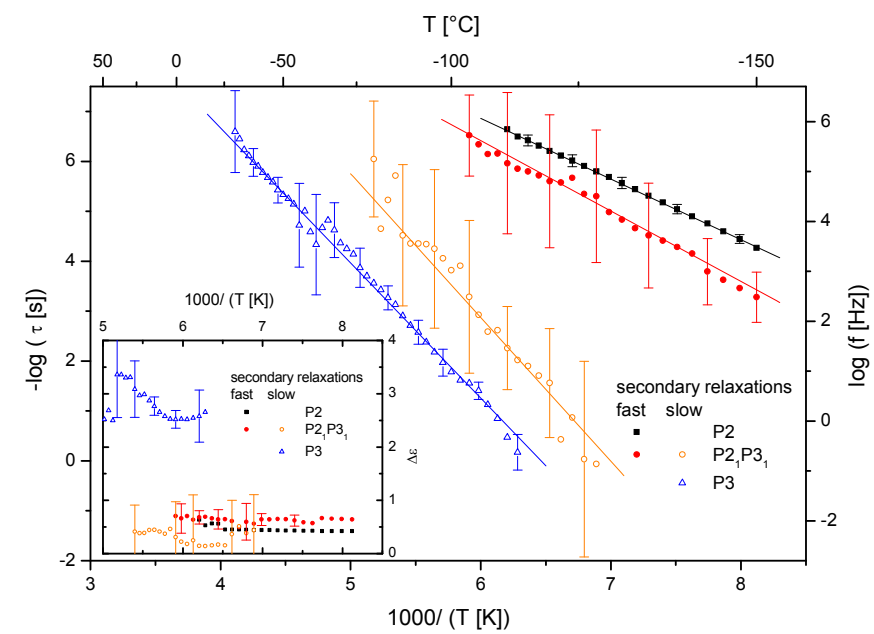

Fig. 6 Mean relaxation time $\tau$ as a function of inverse temperature of the secondary relaxations of the polymers $\mathbf{P} \mathbf{2}, \mathbf{P} \mathbf{2}_{\mathbf{1}} \mathbf{P} \mathbf{3}_{\mathbf{1}}$ and $\mathbf{P} \mathbf{3}$. Inset: Dielectric relaxation strength $\Delta \varepsilon$ of the secondary relaxations of the polymers $\mathbf{P 2}$, $\mathbf{P} \mathbf{2}_{1} \mathbf{P} \mathbf{3}_{1}$ and $\mathbf{P} \mathbf{3}$ as a function of inverse temperature. The error bars indicate the uncertainty of the fit parameters; in the case of $\mathbf{P} \mathbf{2}_{\mathbf{1}} \mathbf{P} \mathbf{3}_{\mathbf{1}}$, the uncertainty in $\tau$ and $\Delta \varepsilon$ is larger due to the proximity of these two processes.

Since the $\mathrm{CN}$ end groups have a much larger dipole moment compared to the methyl groups, the former are expected to show much stronger dielectric relaxation strength. This is indeed the case as verified by the dielectric relaxation strength of the secondary relaxations of $\mathbf{P 2}$ and $\mathbf{P 3}$ (inset of Fig. 6).

However, in polymer $\mathbf{P} \mathbf{2}_{\mathbf{1}} \mathbf{P} \mathbf{3}_{\mathbf{1}}$ the relaxation strength of the faster secondary relaxation (assigned to the methyl-terminated side chains) exceeds that one of the slower relaxation although the number of both types of end groups is balanced. The reason for this is not clear but may be related to the relatively broad relaxation peaks which complicate the precise determination of the relaxation strength. Further, a mutual influence of the two different types of end groups leading to changes in the effective dielectric relaxation strength of each process may play a role.

Beyond the relaxation processes, also the conductivity contribution has been analyzed in the dielectric loss spectra to extract the DCconductivity $\sigma_{D C}$ (Supp. Inform.). The temperature dependence of $\sigma_{D C}$ in $\mathbf{P 2}, \mathbf{P} \mathbf{2}_{\mathbf{1}} \mathbf{P} \mathbf{3}_{\mathbf{1}}$ and $\mathbf{P} \mathbf{3}$ is shown in the inset of Fig. 7. A scaling with respect to $T_{g}$ unravels a quite similar thermal activation (Fig. 7) which suggests a mechanism of charge transport assisted by the glassy dynamics of the polymer matrix (also supported by the VFT 
temperature dependence of $\sigma_{D C}$ ). Remarkable is also the fact that from $\mathbf{P 2}$ to $\mathbf{P} \mathbf{3} \sigma_{D C}$ increases by 4 to 5 orders of magnitude. This pronounced and systematic increase also indicates an origin in the chemical structure of the polymers rather than impurity.

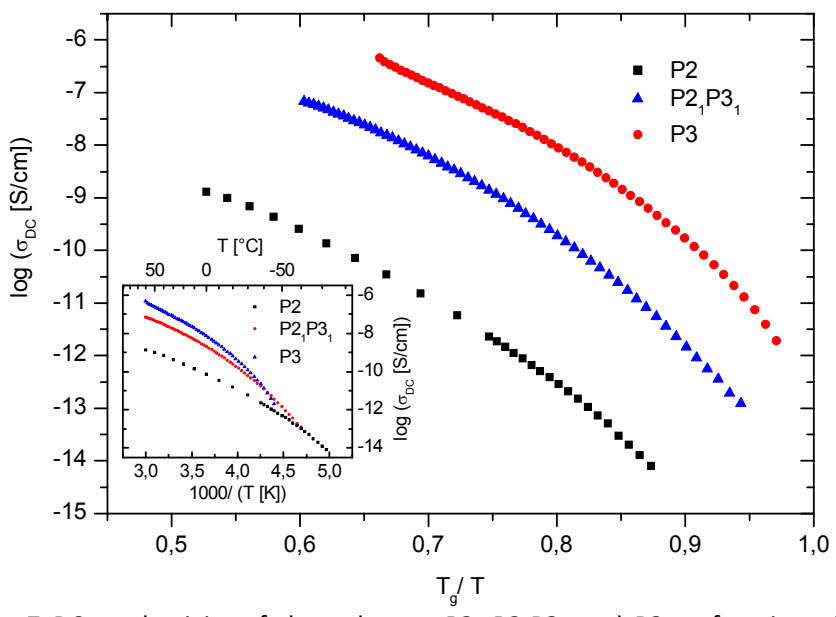

Fig. $\mathbf{7}$ DC-conductivity of the polymers $\mathbf{P} \mathbf{2}, \mathbf{P} \mathbf{2}_{\mathbf{1}} \mathbf{P} \mathbf{3}_{\mathbf{1}}$ and $\mathbf{P} \mathbf{3}$ as function of absolute inverse temperature (inset) and rescaled with respect to the corresponding calorimetric glass transition temperature $T_{g}$. The experimental uncertainty is smaller than the symbol size.

The dielectric properties at room temperature were investigated for all samples $\mathbf{P 2}, \mathbf{P} \mathbf{3}$ and $\mathbf{P} \mathbf{2}_{\mathbf{x}} \mathbf{P} \mathbf{3}_{\mathbf{y}}$ in a frequency range of $0.05 \mathrm{~Hz}$ to 1 $\mathrm{MHz}$ (Fig. S14). Table 2 shows the amount of methanol that contaminate the samples (NMR), while Table 1 summarizes the $T_{g}$, the $\varepsilon^{\prime}$, the predicted static dielectric permittivity $\left(\varepsilon_{s}\right)$ calculated according to Onsager equation, and conductivity at $1 \mathrm{kHz}$ for all prepared polymers. A linear increase in the permittivity with increasing the proportion of nitrile groups in polymers was observed from $\varepsilon^{\prime}=4.7$ for polymer $\mathbf{P 2}$ to a maximum $\varepsilon^{\prime}=18.4$ for $\mathbf{P 3}$. The increase in the permittivity for $\mathbf{P 2}$ to $\varepsilon^{\prime}=4.7$ as compared to regular polydimethylsiloxane is due to the presence of the thioether groups. The values of the permittivity are constant over a broad frequency range from $10^{2} \mathrm{~Hz}$ to $10^{6} \mathrm{~Hz}$, but increase very much at low frequencies due to electrode polarization. An increase in conductivity with increasing the proportion of nitrile groups was observed, but the conductivity values remained in the limits of the dielectric materials.

The Onsager formula (Equation 1), which is valid for polar, non-associating liquids, can be used to calculate the molecular dipole moment $(\mu)$ or the static dielectric permittivity $\left(\varepsilon_{s}\right)$ if either one is known. ${ }^{23}$

$$
\varepsilon_{S}-\varepsilon_{\infty}=\frac{1}{3 \varepsilon_{0}} F \frac{\mu^{2}}{k_{b} T} \frac{N}{V}
$$

where $\varepsilon_{\infty}$ is the high frequency permittivity $\left(\varepsilon_{\infty}=n^{2}\right), N / V$ is the number of dipoles per unit volume, $T$ is the temperature, $\varepsilon_{0}$ the vacuum permittivity, and $k_{b}$ the Boltzmann constant. A correction factor $(F)$ is introduced (Equation 2), which corresponds to the internal field erected by adjacent dipoles.

$$
F=\frac{\varepsilon_{s}\left(\varepsilon_{\infty}+2\right)^{2}}{3\left(2 \varepsilon_{s}+\varepsilon_{\infty}\right)}
$$

The high frequency permittivity values at the lowest temperature $\left(\varepsilon_{\infty}\right)$ were used for the calculations: $\varepsilon_{\infty}=2.9$ for $\mathbf{P 2}$, and $\varepsilon_{\infty}=3.5$ for P3. Dipole moments values of $\mu=1.28 \mathrm{D}$ and $\mu=2.78 \mathrm{D}$ were calculated for the $x$ and $y$ repeat units, respectively. We note that the obtained values for the dipole moments (calculated at a temperature of $22{ }^{\circ} \mathrm{C}$ ) are rather large and thus it has to be expected that the respective molecular entities exhibit a pronounced dipolar interaction. Consequently, the investigated materials do not fulfil the assumptions underlying Equation 1 and the calculated dipole moments represent approximations. An extension of this formula which considers correlations between dipoles is the Kirkwood/Froehlich factor. Its practical computation, however, requires detailed knowledge of both the spatial distribution and the coupling of the correlated dipoles. This is, for polymeric materials, not yet possible since a huge number of dipoles are already coupled by the fact that they belong to the same molecule. As a consequence, Equation 1 also fails to correctly describe the temperature dependence of the dielectric strength (Fig. 6, inset) since the coupling bears further temperature dependence.

Despite these principle obstacles, one can use Equation 3 to calculate the permittivity of $\mathbf{P} \mathbf{2}_{\mathbf{x}} \mathbf{P} \mathbf{3}_{\mathbf{y}}$ :

$$
\frac{\vartheta_{\text {thiol }}\left(\varepsilon_{S}-\varepsilon_{\infty, \text { thiol }}\right) 3 \varepsilon_{S}}{2 \varepsilon_{S}+\varepsilon_{\infty, \text { thiol }}}+\frac{\vartheta_{D P}\left(\varepsilon_{S}-\varepsilon_{\infty, D P}\right) 3 \varepsilon_{S}}{2 \varepsilon_{S}+\varepsilon_{\infty, D P}}=\frac{N_{\text {thiol }}\left(\mu_{\text {thiol }}\right)^{*}}{3 k T \varepsilon_{0}}+\frac{N_{D P}\left(\mu_{D P}{ }^{*}\right)^{2}}{3 k T \varepsilon_{0}}
$$

which is the extension of the Onsager formula to two components where $\rho$ denotes the density of the polymers $\mathbf{P 2}$ and $\mathbf{P 3}(\rho=1.032$ $\mathrm{g} / \mathrm{cm}^{3}$ and $\rho=1.164 \mathrm{~g} / \mathrm{cm}^{3}$, respectively). Since the dipole moments calculated above were obtained without considering the Kirkwood/Froehlich factor the latter is effectively contained in their values. Hence, inserting these values in Equation 4 automatically adds the contribution of the correlation between the dipoles. Fig. 8 shows the calculated and the measured values for the $\varepsilon_{s}$ as a function of the nitrile content. A liniear increase in $\varepsilon_{s}$ with increasing the nitrile content was observed in both cases.

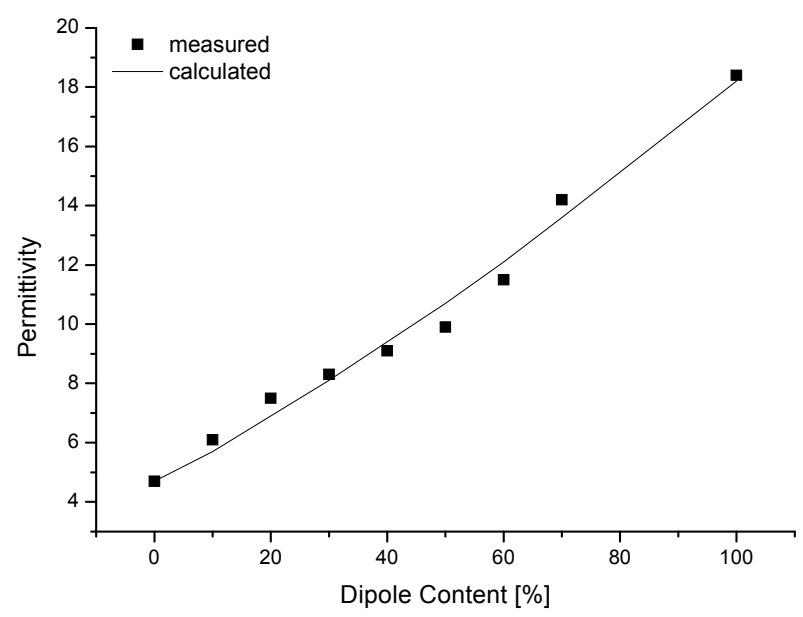

Fig. 8 Variation of static dielectric permittivity $\left(\varepsilon_{s}\right)$ of $\mathbf{P} \mathbf{2}, \mathbf{P} \mathbf{3}$, and $\mathbf{P} \mathbf{2}_{\mathbf{x}} \mathbf{P} \mathbf{3}_{\mathbf{y}}$ as function of polar nitrile content: experimental ( $\mathbf{\square})$ and predicted according 
to Onsager equation (continuous line) for a frequency of $1 \mathrm{kHz}$ and a temperature of $22^{\circ} \mathrm{C}$.

Table 1 The $T_{g}$, and the permittivity and conductivity of polymers $\mathrm{P} 2, \mathrm{P} 3$ and $\mathrm{P} 2{ }_{\mathrm{x}} \mathrm{P} 3_{\mathrm{y}}$ at $1 \mathrm{kHz}$ and $22^{\circ} \mathrm{C}$.

\begin{tabular}{|c|c|c|c|c|c|}
\hline Sample & $T_{g}\left[{ }^{\circ} \mathrm{C}\right]$ & $\begin{array}{c}C_{p} \\
{\left[\mathrm{~J} /\left(\mathrm{g}^{*} \mathrm{~K}\right)\right]}\end{array}$ & $\varepsilon^{\prime}$ & $\varepsilon_{s}^{\text {a) }}$ & $\sigma[\mathrm{S} / \mathrm{cm}]$ \\
\hline P2 & -97.5 & 0.395 & 4.7 & 4.7 & $2.42 \times 10^{-12}$ \\
\hline$P 2_{9} P_{3}$ & -91.6 & 0.252 & 6.1 & 5.7 & $1.64 \times 10^{-11}$ \\
\hline $\mathrm{P2}_{8} \mathrm{P3}_{\mathbf{2}}$ & -86.7 & 0.290 & 7.5 & 6.9 & $4.22 \times 10^{-11}$ \\
\hline $\mathrm{P}_{7} \mathrm{P}_{3}$ & -81.5 & 0.266 & 8.3 & 8.1 & $1.93 \times 10^{-10}$ \\
\hline $\mathrm{P2}_{6} \mathrm{P}_{4}$ & -75.5 & 0.381 & 9.1 & 9.4 & $2.19 \times 10^{-10}$ \\
\hline $\mathbf{P 2}_{1} \mathbf{P B}_{1}$ & -72.1 & 0.381 & 9.9 & 10.7 & $4.50 \times 10^{-10}$ \\
\hline $\mathrm{P2}_{4} \mathrm{P3}_{6}$ & -66.6 & 0.329 & 11.5 & 12.1 & $7.30 \times 10^{-10}$ \\
\hline $\mathrm{P2}_{3} \mathrm{P}_{7}$ & -61.6 & 0.359 & 14.2 & 13.6 & $1.73 \times 10^{-9}$ \\
\hline P3 & -52.6 & 0.432 & 18.4 & 18.2 & $1.84 \times 10^{-7}$ \\
\hline
\end{tabular}

${ }^{\text {a) }}$ The $\varepsilon_{s}$ was calculated using the Onsager equation 1.

1.2.

First preliminary experiments on P3 show that such polymer, despite of its high content of polar nitrile groups, can be crosslinked to a robust elastomer with low elastic modulus $\left(Y_{50 \%}=56\right.$ $\mathrm{kPa}$ ) and strain at break as high as $250 \%$ (Fig. S15 and S16). Further investigations are on the way regarding the optimization of the mechanical properties of this elastomer and its use as a dielectric in electromechanical actuators.

\section{Conclusions}

A series of polysiloxanes containing different proportions of polar nitrile groups were prepared by using thiol-ene photoaddition of thiols 2,3 or a mixture of $2 / 3$ to a high molecular weight P1. A quantitative conversion of the vinyl groups of $\mathbf{P} \mathbf{1}$ occurs within few min. The resulting polymers were characterized by ${ }^{1} \mathrm{H}$ and ${ }^{13} \mathrm{C} N \mathrm{NM}$, IR, GPC, DSC, TGA, and by Broadband Dielectric Spectroscopy (BDS). The latter reveal in a broad spectral and temperature range the segmental relaxation of the siloxane backbone; the scaling with $T_{g}$ verifies that this is the structural relaxation underlying glassy dynamics. Another process with non-Arrhenius type thermal activation which merges with the relaxation of the siloxane segments at $T_{g}$ could not yet be assigned doubtlessly. Two secondary relaxations, a fast and a slow one, can be assigned to local motion of the two types of side chain end groups (methyl and nitrile groups, repsectively). This is evident from the differences in the dynamics (nitrile groups relax slower due to stronger interactions), in the activation energies (larger activation energy in nitrile groups due to stronger interactions) and in the dielectric relaxation strength (larger relaxation strength in nitrile groups due to larger dipole moment). The latter also causes a linear increase in permittivity with increasing the proportion of nitrile groups in the polymers from $\varepsilon^{\prime}$ $=4.7$ for $\mathbf{P 2}$, to $\varepsilon^{\prime}=9.9$ for $\mathbf{P} \mathbf{2}_{\mathbf{1}} \mathbf{P} \mathbf{3}_{1}$, and $\varepsilon^{\prime}=18.4$ for $\mathbf{P 3}$. First preliminary experiments show that the cross-linking of the hydroxyl end-groups with which the polymers are functionalized is possible and allows formation of elastomeric materials. Therefore, this work opens up the access to a new class of elastomeric materials with dielectric properties that can be fine-tuned. Work in this direction is on the way.

\section{Experimental}

\section{Materials}

Unless otherwise stated, all chemicals were reagent grade and used as received. TMAH $\times 5 \quad \mathrm{H}_{2} \mathrm{O}$ and 1,3,5,7-tetravinyl-1,3,5,7tetramethylcyclotetrasiloxane $\left(\mathrm{V}_{4}\right)$ were purchased from $A B C R .2,2-$ dimethoxy-2-phenylacetophenone (DMPA), butanethiol, NaSH, tetrakis(dimethylsiloxy)silane, dibutyltindilaurate, benzene, toluene and acrylonitrile were purchased from Aldrich while methanol, dichloromethane $\left(\mathrm{CH}_{2} \mathrm{Cl}_{2}\right)$, hexane, tetrahydrofurane (THF), and ethyl acetate were purchased from VWR. Benzene was dried over sodium using benzophenone indicator. Thiol $\mathbf{3}^{17}$ and polymer $\mathbf{P} \mathbf{1}^{18}$ (GPC: $M_{n}=72 \mathrm{kDa}, M_{w}=169 \mathrm{kDa}, D=2.4$ ) were prepared according to the literature.

\section{Methods}

${ }^{1} \mathrm{H},{ }^{13} \mathrm{C}$ and ${ }^{29} \mathrm{Si}$ NMR spectra were recorded on a Bruker Avance III 400 NMR spectrometer using a $5 \mathrm{~mm}$ BBO Prodigy ${ }^{\mathrm{TM}}$ CryoProbe at $400.18,100.63$ and $79.50 \mathrm{MHz}$, respectively. Chemical shifts $(\delta)$ in ppm are calibrated to residual solvent peaks $\left(\mathrm{CDCl}_{3}: \delta=7.26\right.$ and $77.16 \mathrm{ppm})$. IR spectra were recorded on a Bruker Tensor 27 ATR FT-IR. GPC was measured using an Agilent 1100 Series HPLC (Columns: serial coupled PSS SDV $5 \mathrm{u}, 100 \mathrm{~A}$ and PSS SDV $5 \mathrm{u}, 1000 \mathrm{~A}$, Detector: DAD, $235 \mathrm{~nm}$ and $360 \mathrm{~nm}$; refractive index) with THF as mobile phase. Calibration was done with PDMS standards and toluene was used as internal standard. The thermogravimetric analysis (TGA) was conducted with a Perkin Elmer TGA7 at a heating rate of $20^{\circ} \mathrm{C} \mathrm{min}^{-1}$ under a nitrogen gas flow. Differential scanning calorimetry (DSC) investigations were undertaken on a Pyris Diamond DSC (Perkin Elmer USA) instrument under a nitrogen flow $\left(50 \mathrm{~mL} \mathrm{~min}{ }^{-1}\right.$ ), in aluminium crucibles shut with pierced lids and using a $10 \mathrm{mg}$ sample mass. Photoreactions were conducted with a UVAHAND 250 GS H1 mercury vapour UV lamp from Hönle UV technology. Density measurements were done using a Mettler Toledo XP 504 balance.

For the broadband dielectric spectroscopy (BDS) measurements a high impedance Alpha Analyzer combined with a Quatro temperature controller (both from Novocontrol) has been employed to cover a broad frequency and temperature range from $0.1 \mathrm{~Hz}$ to $1 \mathrm{MHz}$ and from -150 to $60^{\circ} \mathrm{C}$, respectively. The samples were annealed at $60^{\circ} \mathrm{C}$ for about $5 \mathrm{~h}$ to remove any residual solvents, before isothermal measurements were performed. During the whole annealing and measurement procedure the samples were kept in dry nitrogen atmosphere. Two stainless steel discs with a diameter of $20 \mathrm{~mm}$ served as electrodes which were separated by three glass fibers with a diameter of $\sim 50 \mu \mathrm{m}$ (the exact diameter of the fibers has been determined with a micrometer screw before each measurement with an uncertainty of $1 \mu \mathrm{m})$.

\section{General procedure for the synthesis of $\mathrm{P2}_{x} \mathrm{P3}_{\mathrm{y}}$}


To a solution of $\mathbf{P 1}(8 \mathrm{~g})$ in THF $(200 \mathrm{ml})$, different $\mathrm{x}$ :y ratios of thiols 2:3 and DMPA (0.01 eq to vinyl) were added. Table 2 includes the amount of substances used for the synthesis of $\mathbf{P} \mathbf{2}, \mathbf{P} \mathbf{3}$, and $\mathbf{P} \mathbf{2}_{\mathbf{x}} \mathbf{P} \mathbf{3}_{\mathbf{y}}$. The reaction mixture was set under argon and irradiated with UVlight for $20 \mathrm{~min}$. It was then concentrated under vacuum to about $50 \mathrm{ml}$. To this concentrated solution, methanol was added whereupon the polymer precipitates. The polymer was dissolved in toluene and again precipitated with methanol. The dissolution/precipitation process was repeated three times. The polymers were then dried under $\mathrm{HV}$ at $60^{\circ} \mathrm{C}$ to give yellowish highly viscous liquids. The characterization of polymers $\mathbf{P 2}, \mathbf{P} \mathbf{2}_{\mathbf{x}} \mathbf{P} \mathbf{3}_{\mathbf{y}}$, and $\mathbf{P} \mathbf{3}$ is given in Supporting Information.

Table 2 The amounts of reagents and reaction conditions used for the synthesis of $\mathbf{P} \mathbf{2}, \mathbf{P} \mathbf{3}$, and $\mathbf{P} \mathbf{2}_{\mathbf{x}} \mathbf{P} \mathbf{3}_{\mathbf{y}}$ and their composition, molecular weights and distributions, as well as the residual methanol left in the samples (Imp).

\begin{tabular}{|c|c|c|c|c|c|c|c|c|c|c|c|c|}
\hline \multirow[t]{2}{*}{ Sample } & \multicolumn{2}{|c|}{$P 1^{\text {a) }}$} & \multicolumn{2}{|c|}{2} & \multicolumn{2}{|c|}{3} & \multicolumn{2}{|c|}{$\mathrm{mol} \%$} & \multirow{2}{*}{$\begin{array}{c}M_{w} \\
{[\mathrm{kDa}]}\end{array}$} & \multirow{2}{*}{$\begin{array}{c}M_{n} \\
{[\mathrm{kDa}]}\end{array}$} & \multirow[t]{2}{*}{ PDI } & \multirow{2}{*}{$\begin{array}{l}\operatorname{lmp}^{\mathrm{d})} \\
{[\mathrm{w} \%]}\end{array}$} \\
\hline & {$[\mathrm{g}]$} & {$[\mathrm{mmol}]$} & [g] & {$[\mathrm{mmol}]$} & {$[\mathrm{g}]$} & {$[\mathrm{mmol}]$} & $\mathrm{CN}^{\mathrm{b})}$ & $\mathrm{CN}^{\mathrm{c})}$ & & & & \\
\hline P2 & 7.98 & 92.7 & 13 & 144.2 & - & - & 0 & 0 & 219 & 97 & 2.2 & 0.35 \\
\hline $\mathbf{P 2}_{9} \mathbf{P 3}_{1}$ & 8.08 & 93.7 & 15 & 166.4 & 1.62 & 18.6 & 10 & 11 & 258 & 99 & 2.6 & 0.23 \\
\hline $\mathbf{P 2}_{8} \mathbf{P 3}_{2}$ & 8.07 & 93.6 & 13.3 & 147.7 & 3.22 & 36.9 & 20 & 20.6 & 275 & 100 & 2.7 & 0.08 \\
\hline $\mathrm{P2}_{7} \mathrm{P}_{3}$ & 8.01 & 93.6 & 11.73 & 130.1 & 4.83 & 55.4 & 30 & 30.6 & 279 & 98 & 2.9 & 0.52 \\
\hline $\mathrm{P2}_{6} \mathrm{P3}_{4}$ & 8.01 & 92.9 & 10.05 & 111.4 & 6.50 & 74.6 & 40 & 42.7 & 258 & 93 & 2.8 & 0.16 \\
\hline$P 2_{1} P_{3}$ & 7.99 & 92.7 & 8.42 & 93.3 & 8.15 & 93.6 & 50 & 48 & 294 & 97 & 3.0 & 0.09 \\
\hline $\mathrm{P2}_{4} \mathrm{P3}_{6}$ & 8.04 & 93.4 & 6.69 & 74.1 & 9.8 & 112.4 & 60 & 58 & 294 & 95 & 3.1 & 0.03 \\
\hline $\mathrm{P2}_{3} \mathrm{P3}_{7}$ & 7.99 & 92.7 & 5.02 & 55.6 & 11.32 & 129.8 & 70 & 70 & 242 & 86 & 2.8 & 0.11 \\
\hline P3 & 20.0 & 232.2 & - & - & 40.5 & 464.5 & 100 & 100 & 124 & 52 & 2.4 & 0.12 \\
\hline
\end{tabular}

${ }^{\text {a) }} 0.01$ eq DMPA to vinyl were used; ${ }^{\text {b) }}$ theoretical mol\% CN; ${ }^{c)}$ mol\% $\mathrm{CN}$ as calculated from the ${ }^{1} \mathrm{H}$ NMR spectra; ${ }^{\mathrm{d})}$ the amount of residual methanol in the polymers as determined by ${ }^{1} \mathrm{H}$ NMR.

\section{Acknowledgements}

We gratefully acknowledge the financial support of Swiss National Science Foundation and UEFISCDI, under the Swiss- Romanian Cooperation Program, grant No. IZERZO_142215 / 1 (10 / ROCH/RSRP/01.01.2013), Swiss Federal Laboratories for Materials Science and Technology (Empa, Dübendorf), and COST (European Cooperation in Science and Technology) in the framework of ESNAM (European Scientific Network for Artificial Muscles) - COST Action MP1003. DMO would also like to thank Prof. J. Feast from Durham University for helpful discussions. We also like to acknowledge $\mathrm{B}$. Fischer for the TGA and DSC measurements, and D. Rentsch for helping with the NMR measurements. The NMR hardware was partially granted by the Swiss National Science Foundation (SNFS, grant No. 150638).

Author Contributions

S.D. performed the synthesis of all the compounds. M.T. conducted broadband dielectric spectroscopy measurements at different temperatures. M.T. and F.K. did the interpretation of the dielectric spectra and wrote this part of the manuscript. S.Y.K conducted the dielectric measurements at room temperature. C.R. conducted the DSC measurements and F.N. read the manuscript and gave scientific input. D.M.O. initiated this research, designed the polymers, coordinated the research and wrote the manuscript with input from all authors. All authors have given approval to the final version of the manuscript.

\section{Notes and references}

1 W. D. Callister, Materials Science and Engineering and Introduction, John Wiley \& Sons, Inc. 2003.
2 F. Carpi, D. De Rossi, R. Kornbluh, R. Perline, P. SommerLarsen, Dielectric elastomers as electromechanical transducers, Elsevier: Amsterdam, 2008.

3 P. Brochu, Q. Pei, Macromol. Rapid Commun. 2010, 31, 1036; J. Biggs, K. Danielmeier, J. Hitzbleck, Angew. Chem. Int. Ed. 2013, 52, 9409-9421.

4 M. Molberg, D. Crespy, P. Rupper, F. Nüesch, J.-A. E. Månson, C. Löwe, D. M. Opris, Adv. Funct. Mater. 2010, 20, 32803291.

5 S. Sherratt, R. Bringer, L. L. Ferstandig, L. E. Wolinski, W. S. Barnhart, N. T. Hall, The chemistry and technology of fluorine. Kirk-Othmer encyclopedia of chemical technology, Wiley, New York, 1966.

6 W. J. Feast, M. Gimeno, E. Khosravi, Polymer, 2003, 44, 6111-6121.

7 M.F. Bukhina, S.K. Kurlyand, Low-Temperature Behaviour of Elasomers, Brill, Leiden, 2007.

8 J. M. Davies, R. F. Miller, W. F. Busse, J. Am. Chem. Soc. 1941, 63, 361.

9 J. Feast, M. Gimeno, R. Cochlin, E. Khosravi, New Functional Polymers via ROMP: An Approach to Electrostrictive Polymers, presentation at NASA; G. R. Davies, H. V. St A. Hubbard, I. M. Ward, W. J. Feast, V. C. Gibson, E. Khosravi, E. L. Marshall, Polymer, 1995, 36, 235-243.

10 D. M. Opris, Material development for dielectric elastomer actuators, presentation at EAP Winterschool, January, 2010, Ascona, Switzerland; D. M. Opris, Material development for dielectric elastomer actuators, presentation at Danforss PolyPower, June, 2010, Nordborg, Denmark.

11 C. Racles, M. Cazacu, D. M. Opris, EuroEAP, 1st Int. Conf. on Electromechanically Active Polymer (EAP) Transducers \& Artificial Muscles (Pisa) 2011, http://win.euroeap.eu/public/2.3.8.pdf; H. Böse, D. Uhl, R. Rabindranath, Proc SPIE, 2012, 8340: 83402E(1-10); S. Risse, B. Kussmaul, H. Krüger, G. Kofod, Adv. Funct. Mater. 2012, 22, 3958-3962; B. Kussmaul, S. Risse, G. Kofod, R. Wache, M. Wegener, D. N. McCarthy, H. Krüger, R. Gerhard, Adv. Funct. Mater. 2011, 21, 4589-4594; C. Racles, M. Cazacu, B. Fischer, D. M. Opris, Smart Mater. Struct. 2013, 22, 104004; F. B. 
Madsen, L. Yu, A. E. Daugaard, S. Hvilsted, A. L. Skov, RSC Advances, 2015, 5, 10254

12 B. Marciniec, Advances in Silicon Science, Springer, Dordrecht, 2009.

13 H. C. Kolb, M. G. Finn, K. B. Sharpless, Angew. Chem. Int. Ed., 2001, 40, 2004-2021.

14 C. Racles, M. Alexandru, A. Bele, V. E. Musteata, M. Cazacu, D. M. Opris, RSC Adv., 2014, 4, 37620-37628.

15 R. K. Iha, K. L. Wooley, A. M. Nyström, D. J. Burke, M. J. Kade, C. J. Hawker, Chem Rev. 2009, 109, 5620-5686.

16 F. B. Madsen, I. Javakhishvili, R. E. Jensen, A. E. Daugaard, S. Hvilsted, A. L. Skov, Polym. Chem. 2014, 5, 7054-7061.

17 G. P. Miller, A. P. Silverman, E. T. Kool, Bioorg. Med. Chem., 2008, 16, 56-64.

18 D. W. Kang, B. C. Lee, Polymer, 2004, 28, 143-148; M. Circu, Y. S. Ko, F. A. Nüesch, A. G. Christian, D. M. Opris, Macromol. Mater. Eng., 2014, 299, 1126-1133.

19 L. Herczynska, L. Lestel, S. Boileau, J. Chojnowski, S. Polowinski, Eur. Pol. J. 1999, 35, 1115-1122; B. Boutevin, Y. Pietrasanta, L. Sarraf, J. Fluor. Chem. 1986, 31, 425; R. Dorigo, A.-M. Garnault, D. Teyssie, S. Boileau, Integrated Fundam. Polymer Science Technology 2, Elsevier, London, 1987; K. Rozga-Wijas, K. J. Chojnowski, T. Zundel, S. Boileau, Macromol. 1996, 29, 2711; K. Rozga-Wijas, J. Chojnowski, S. Boileau, J. Polym. Sci. A: Polym. Chem. 1997, 35, 879.

20 H. Adachi, K. Adachi, Y. Ishida, T. Kotaka, J. Polym. Sci.: Polym. Phys. Ed. 1974, 17, 851; K. U. Kirst, F. Kremer, V. M. Litvinov, Macromol., 1993, 26, 975-980.

21 S. Havriliak, S. Negami, Polymer, 1967, 8, 161.

22 H. Vogel, Physikalische Zeitschrift, 1921, 22, 645; G. S. Fulcher, J. Am. Chem. So., 1925, 8, 339; G. Tammann, G. Hesse, Zeitschrift für anorganische und allgemeine Chemie, 1926, 156, 245.

23 L. Onsager, J. Am. Chem. Soc., 1936, 58, 1486; F. Kremer, A. Schönhals, Broadband dielectric spectroscopy, Springer, 2002. 